\title{
Historical Remarks on Suslin's Problem
}

\author{
Akihiro Kanamori ${ }^{1}$
}

The work of Stanley Tennenbaum in set theory was centered on the investigation of Suslin's Hypothesis (SH), to which he made crucial contributions. In 1963 Tennenbaum established the relative consistency of $\neg \mathrm{SH}$, and in 1965, together with Robert Solovay, the relative consistency of SH. In the formative period after Cohen's 1963 founding of forcing when set theory was transmuting into a modern, sophisticated field of mathematics, this work on $\mathrm{SH}$ exhibited the power of forcing for elucidating a classical problem of mathematics and stimulated the development of new methods and areas of investigation.

$\S 1$ discusses the historical underpinnings of SH. $\S 2$ then describes Tennenbaum's consistency result for $\neg \mathrm{SH}$ and related subsequent work. $\S 3$ then turns to Tennenbaum's work with Solovay on SH and the succeeding work on iterated forcing and Martin's Axiom. To cast an amusing sidelight on the life and the times, I relate the following reminiscence of Gerald Sacks from this period, no doubt apocryphal: Tennenbaum let it be known that he had come into a great deal of money, $\$ 30,000,000$ it was said, and started to borrow money against it. Gerald convinced himself that Tennenbaum seriously believed this, but nonetheless asked Simon Kochen about it. Kochen replied, "Well, with Stan he might be one per-cent right. But then, that's still $\$ 300,000$."

\section{$\S 1$. Suslin's Problem}

In 1920, at the end of the first volume of Fundamenta Mathematicae there appeared a list of problems with one attributed to Mikhail Suslin [1920], a problem that would come to be known as Suslin's Problem. After the reunification of Poland in 1918, there was a deliberate decision by its aspiring mathematicians to focus on set theory and related areas and to bring out a new journal to promote international research in this area. ${ }^{2}$ This was the origin of Fundamenta Mathematicae, which became the main conduit of scholarship in 'fundamental mathematics' during the 1920s and 1930s. That first list of problems had to do with possible consequences of the Continuum Hypothesis $(\mathrm{CH})$ or issues in the emerging descriptive set theory. These problems would be solved, but by contrast Suslin's Problem would grow in significance through its irresolution.

Georg Cantor, the founder of set theory, had famously characterized the ordertypes of the rationals and reals in his Beiträge [1895], his mature presentation of his theory of the transfinite. The ordering of the reals is that unique dense linear ordering with no endpoints which is order-complete (i.e. every bounded set has a least upper bound ${ }^{3}$ ) and separable (i.e. has a countable dense subset). Suslin's Problem asks whether this last condition can be weakened to the countable chain condition (c.c.c.): every disjoint family of open intervals is countable. Although Suslin himself did not hypothesize it, the affirmative answer has come to be known as Suslin's Hypothesis (SH). For a dense linear order, deleting endpoints and

\footnotetext{
1 This is an expanded version of an invited address given at a memorial conference commemorating the life and work of Stanley Tennenbaum held at the Graduate Center of the City University of New York on 7 April 2006.

2 See Kuratowski [1980] and Kuzawa [1968].

3 Cantor had every fundamental (Cauchy) sequence being complete, but this has to be mediated by the Axiom of Choice.
} 
taking the (Dedekind) completion does not affect the c.c.c. or separability properties. So, the hypothesis could be simply stated as:

Every (infinite) dense linear ordering satisfying the c.c.c. is separable.

Suslin's Problem would be the first anticipation of the study of chain conditions in general topology, and as such it displays a remarkable foresight. Suslin himself was a wunderkind who after finding a mistake in a paper of Lebesgue formulated the analytic sets and established [1917] fundamental results about them: a set of reals is Borel exactly when it and its complement are analytic, and there is an analytic set which is not Borel. These seminal results considerably stimulated the Soviet and Polish schools in descriptive set theory, and some of the problems on that first Fundamenta list concerns analytic sets. ${ }^{4}$ [1917] was to be Suslin's sole publication, ${ }^{5}$ for he succumbed to typhus in the 1919 Moscow epidemic at the age of 25. Until the early 1970s one sees the "Souslin" from Suslin [1920]; this is the French transliteration, for Fundamenta at first adopted French as the lingua franca.

Recapitulating the mathematical experience, it is hard to see how to go from the rather amorphous countable chain condition to a countable dense subset. To compare, Cantor had first formulated $\mathrm{CH}$ as the loose assertion that there is no strictly intermediate power between that of the natural numbers and that of the continuum. Canter then developed the transfinite numbers and converted $\mathrm{CH}$ to the positive, existence assertion that there is a bijection between the continuum and the countable ordinals, and thereafter tried to exploit analogies between increasing convergent sequences of reals and such sequences of countable ordinals. With Suslin's Problem, in the several decades after its articulation what interest there was in it became focused on $\neg \mathrm{SH}$, a positive, existence assertion which became characterized in a perspicuous form which suggested possibilities for establishing it. Both mathematically and historically, when "Suslin's Hypothesis" came into use it should thus have arguably have referred to $\neg \mathrm{SH}$.

A tree is a partially ordered set $\left\langle T,<_{T}\right\rangle$ with a minimum element such that for any $x \in T$ the set $\left\{y \in T \mid y<_{T} x\right\}$ of its $<_{T}$-predecessors is well-ordered by $<_{T}$. The $\alpha$ th level of $T$ consists of those $x \in T$ whose set of $<_{T}$-predecessors has ordertype $\alpha$ under $<_{T}$. The height of $T$ is the least $\alpha$ such that the $\alpha$ th level of $T$ is empty. A chain of $T$ is a linearly ordered subset, and an antichain of $T$ is a subset consisting of pairwise $<_{T}$-incomparable elements. A Suslin tree is a tree of height $\omega_{1}$ with no uncountable chains or antichains.

Trees abound in contemporary set theory as basic combinatorial objects. ${ }^{6}$ The first systematic study of trees was carried out in Djuro Kurepa's Paris thesis [1935] with Fréchet, where several tree and linear order equivalences were derived. Kurepa [1935: 127ff][1936] provided the following characterization, since rediscovered by Edwin Miller [1943] and Wacław Sierpiński [1948]:

$\neg \mathrm{SH}$ iff there is a Suslin tree.

In the forward direction, let $\left\langle S,<_{S}\right\rangle$ be a counterexample to $\mathrm{SH}$, i.e. a dense linear ordering with the c.c.c. but with no countable dense subset. Recursively construct non-

4 See Kanamori [1995] for the emergence of descriptive set theory.

5 Suslin [1920] is an attribution to Suslin. It should be observed that accompanying Suslin [1917] was a note by Nikolai Luzin [1917] in which he credited Suslin with having established that every analytic set has the perfect set property.

6 See Todorčević [1984] for a magisterial account. 
empty open intervals $I_{\alpha}$ for $\alpha<\omega_{1}$ as follows: Let $I_{0}$ be $S$. Given $I_{\beta}$ for $\beta<\alpha$, since the set $E$ of all the endpoints of these intervals is countable, let $I_{\alpha}$ be an interval disjoint from $E$. Then $\left\{I_{\alpha} \mid \alpha<\omega_{1}\right\}$ under reverse inclusion is a Suslin tree.

In the converse direction, let $\left\langle T,<_{T}\right\rangle$ be a Suslin tree. By successively pruning and omitting intermediate nodes, we can assume that: every element has uncountably many successors; different elements at a limit level do not have the same sets of predecessors; and every element has more than one immediate successor. We can further assume, by restricting to the limit levels only, that every element has infinitely many immediate successors. Now, linearly order each level as a dense linear order without endpoints. Then let $S$ consist of the maximal chains ("branches") of $T$, and for $c_{1} \neq c_{2} \in S$, define $c_{1}<_{S} c_{2}$ exactly when at the least level at which they differ, the element in $c_{1}$ precedes the element in $c_{2}$ in that level's linear order. Then $\left\langle S,<_{S}\right\rangle$ is a counterexample to $\mathrm{SH}$.

This tree characterization of $\neg \mathrm{SH}$ eliminated topological considerations from Suslin's Problem and reduced it to a problem of combinatorial set theory. Suslin trees and their generalizations have since played important roles in modern set theory both in providing examples and in promoting the development of set-theoretic methods. Even early on, $\neg \mathrm{SH}$ led to examples in general topology (cf. Rudin [1955]) and played a role analogous to $\mathrm{CH}$ an unproved hypothesis from which significant conclusions were drawn. This incidentally, is further reason why "Suslin's Hypothesis" should be attached to $\neg \mathrm{SH}$. In any case, $\neg \mathrm{SH}$, like $\mathrm{CH}$, would have to await Cohen's invention of forcing for significant elucidation.

\section{$\S 2$. Consistency of $\neg \mathrm{SH}$}

Making the first real breakthrough on Suslin's Problem since its articulation, Tennenbaum [1968] in 1963 established the relative consistency Con(ZFC) implies Con(ZFC $+\neg \mathrm{SH})$. This he did by devising a notion of forcing for adding a Suslin tree. Proceeding in modern vein, Tennenbaum's partial order consists of finite trees $\left\langle t,<_{t}\right\rangle$, where $t \subseteq \omega_{1}$ and $\alpha_{<_{t}} \beta$ implies $\alpha<\beta$, ordered by:

$$
\left\langle t_{1},<_{t_{1}}\right\rangle \leq\left\langle t_{2},<_{t_{2}}\right\rangle \text { iff } t_{1} \supseteq t_{2} \wedge<_{t_{2}}=<_{t_{1}} \cap\left(t_{2} \times t_{2}\right) .
$$

That this partial order has the countable chain condition ${ }^{7}$ can be established by a typical $\Delta$-system argument, and hence forcing with it preserves all cardinals.

For a generic $G$, define

$$
\begin{aligned}
& T=\bigcup\left\{t \mid\left\langle t,<_{t}\right\rangle \in G\right\}, \text { and } \\
& <=\bigcup\left\{<_{t} \mid\left\langle t,<_{t}\right\rangle \in G\right\},
\end{aligned}
$$

$\langle T,<\rangle$ is a tree, of height $\omega_{1}$ by a density argument. Another $\Delta$-system argument establishes that this tree has no uncountable antichains. Finally, this tree cannot have any uncountable chains either, as such a chain would engender an uncountable antichain consisting of "offshoots". Hence, $\langle T,<\rangle$ is a Suslin tree.

To the set theorist of today this consistency result is quite straightforward, and even Tennenbaum once told me that it was merely an "exercise" in forcing. However, it is remarkable that, according to a footnote of Tennenbaum [1968], the work was done in the

7 This of course is in the well-known sense for a forcing partial order. The c.c.c. defined earlier will be consistent with this, if we take the open sets of the topology under the partial order of inclusion. 
summer of 1963. Cohen had just come up with forcing that spring and had established his relative consistency results about the Continuum Hypothesis and the Axiom of Choice. With Solomon Feferman, Robert Solovay, and Azriel Levy, Tennenbaum would be among the first after Cohen who established results with forcing. Moreover, Tennenbaum's notion of forcing was the first to address issues in ZFC other than the sort that Cohen himself addressed, about powers of cardinals, collapsing cardinals, and definability. Tennenbaum expressed gratitude to Georg Kreisel, Anil Nerode, and Dana Scott for pointing out gaps in previous attempts and to Kurt Gödel, who communicated the paper, for simplifying the countable chain condition argument.

Several years later Thomas Jech [1967] independently established the consistency of $\neg \mathrm{SH}$. Working in Prague, he approached the result through Petr Vopěnka's $\nabla$-models. Of note, there was earlier Russian-Eastern European work by Yesenin-Volpin [1954], who had produced a Fraenkel-Mostowski permutation model of $\neg \mathrm{SH}$ with continuum many urelements. Jech's forcing conditions, unlike Tennenbaum's, are countable approximations to a Suslin tree ordered by end-extension. Cardinals are preserved because of the countable closure of the partial order, and that the resulting tree has no uncountable antichains is established by a Skolem hull argument. On the one hand, this argument is more involved than its counterpart in Tennenbaum's proof, but on the other hand, it is Jech's proof that would generalize in the later work on higher cardinality versions of Suslin trees. From the modern perspective it is more natural to consider initial segments of a possible Suslin tree as conditions, but Tennenbaum presumably worked out his approach because of its affinity to Cohen's for violating $\mathrm{CH}$; countable closure of forcing conditions was not used by Cohen, but he evidently used the countable chain condition. Also, Tennenbaum's argument works whether $\mathrm{CH}$ or $\neg \mathrm{CH}$ holds and preserves that state of affairs; Jech's forcing always enforces $\mathrm{CH}$.

The most significant result about $\neg \mathrm{SH}$, one that moreover would have the most potentiality, was the result of Ronald Jensen [1968] that for Gödel's constructible universe $L$, if $V=L$, then there is a Suslin tree. Jensen famously isolated a combinatorial principle $\diamond$ that carried what was needed of the structure of $L$ and showed that $\diamond$ itself implies that there is a Suslin tree. This would not only lead to generalizations to higher cardinals but also begin Jensen's broad investigation of pivotal combinatorial principles holding in $L$, principles that would achieve autonomous status for establishing a wide range of propositions of combinatorial set theory.

There is an underlying similarity between Jech's argument and Jensen's for establishing that the tree has no uncountable antichains. At base there is a common forcing argument for how levels of the tree are to be generically extended. Jech's forcing is actually equivalent to the forcing $Q$ for adding a Cohen subset of $\omega_{1}$ with countable conditions. ${ }^{8} Q$ actually adjoins $\diamond$, and hence by Jensen's result that there is a Suslin tree.

Tennenbaum's forcing is rather like the standard forcing for adding $\aleph_{1}$ reals (with finite conditions). Saharon Shelah [1984] eventually established that adding even one Cohen real adjoins a Suslin tree. This last is a remarkable result, and provides a structured sense to the assertion that one is always very close to having a Suslin tree.

8 And $Q$ is in turn equivalent to the usual forcing for collapsing $2^{\aleph_{0}}$ to $\omega_{1}$, since every condition in $Q$ has $2^{\aleph_{0}}$ many incompatible extensions. 


\section{$\S 3$. Consistency of SH}

Making those moves in set theory that would be the most consequential not only for Suslin's Problem but for the development of forcing in general, Tennenbaum began to investigate the possibility of establishing the consistency of SH. It had been simple enough to add a Suslin tree by forcing, and now there was the difficult prospect of getting a model with no Suslin trees. Tennenbaum saw how "to kill a Suslin tree": That a Suslin tree has no uncountable antichains is exactly the property of the partial order having the countable chain condition, and so forcing with the tree itself generically adds an uncountable chain. In fact, it is easy to see that any uncountable chain actually corresponds to a generic object, and so genericity was intrinsic to having an uncountable chain. Here for the first time was the recasting a combinatorial object of classical mathematics as itself a notion of forcing. But what now? Once a Suslin tree is thus killed it stays dead, but other Suslin trees might have sprung up, and so they too must be killed by iterating the process. This is where the collaboration with Robert Solovay came in.

Robert Solovay above all epitomized this period of great expansion in set theory, with his mathematical sophistication and his fundamental results with forcing, in large cardinals, and in descriptive set theory. Following initial graduate study in differential topology, Solovay focused his energies on set theory after attending a lecture of Cohen's in May 1963. Solovay first extended the independence of $\mathrm{CH}$ by characterizing the possibilities for the size of the continuum, and then generalized Cohen's forcing to arbitrary partial orders and dense sets. He next established his famous Lebesgue measurability result during March-July 1964 (Solovay [1970: 1]). Then with Tennenbaum he worked out the iterated forcing proof of the consistency of SH (Solovay-Tennenbaum [1971:201]). An email letter of 18 February 2006 from Solovay to the author describes the interaction with Tennenbaum and the main features of their proof for Con(ZFC) implies Con $(\mathrm{ZFC}+\mathrm{SH})$; the letter is quoted here with Solovay's permission, verbatim except for a side remark at the first (b).

Aki,

Sorry to have taken so long to get back to your request re Tennenbaum. I hope these comments are not too late.

At one crucial point in the following my memory is not clear and I have tried to reconstruct what happened. I've labeled the reconstruction as speculation in what follows.

I was at the Institute for Advanced Study in Princeton during the year 1964-65. I think Stan was teaching somewhere in Philadelphia at the time. At any rate, he would come up regularly to Princeton to talk about set theory and more specifically his attempt to prove the consistency of Souslin's Hypothesis.

At that time, he had already proved the independence of SH via a forcing argument where the conditions were finite approximations to the Souslin Tree that was to be generically added.

His attempt/plan for the consistency of SH had the following ingredients:

(a) it was to be an iteration in which at each step another Souslin tree would be killed.

(b) The steps in the iteration were to be forcing with Souslin trees. ...

(c) Stan knew that forcing with a Souslin tree killed it and that the forcing was c.c.c.

For much of the year, Stan was trying to prove that the iteration did not collapse cardinals. And he was considering iterations of lengths 2 and 3. My role was to passively listen to his proofs and spot the errors in them. With unjustified prescience, I kept saying that I was worried about "killing the same tree twice". Of course, there now are examples due to Jensen that show that 
forcing with the product of a Souslin tree with itself can collapse cardinals. But these weren't available then.

At one of those meetings one of us (probably Stan) made progress and finally found reasonable conditions under which a two stage iteration did not collapse cardinals.

Somehow this got me seriously thinking about the problem and by the time of our next meeting I had a proof of the theorem. This proof had the following ingredients which were new:

(a) defining a transfinite sequence of forcing notions $P_{\alpha}$ where $P_{\alpha+1}=P_{\alpha} * Q_{\alpha}$.

This involves: (a1) defining the operation $*$ where $P * Q$ is defined if $Q$ is a poset in $V^{P}$;

(a2) defining what to do at limits.

(b) proving that if the component forcings are c.c.c. then the limit forcing is c.c.c.;

(c) exploiting the c.c.c.ness to see that if the length of the iteration has cofinality greater than $\omega_{1}$ then all subsets of $\aleph_{1}$ in the final model appear at some proper intermediate stage;

(d) setting up the bookkeeping so that all Souslin trees are killed.

I admit that, with hindsight, all these things look easy now.

I come now to the speculation as to what was discovered that day that set me to thinking. My guiding principle is that it has to be something which is not utterly trivial.

I think that Stan was looking at the product of two Souslin trees in the initial ground and trying to show the product was c.c.c.

Of course, with this level of generality this is not true. I suspect that the discovery was that if the second tree $T_{2}$ remained Souslin in $V\left[T_{1}\right]$ then the composed forcing was c.c.c.

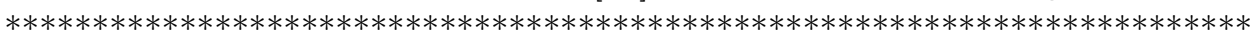

When it came time to write the proof up, I was under the spell of Boolean valued models [which I had just discovered] and asked Stan if I could present the proof in terms of them. He agreed, but subsequently had strong reservations, and the paper was almost not published. [He eventually agreed to its publication with a footnote expressing his reservations about the Boolean valued approach. The footnote is in the published paper.] Whether I would have had the stomach to withdraw the paper from the Annals [it had already been accepted] and rewrite it completely, I don't know. I'm glad it didn't come to that.

I hope these comments are useful to you. As I write them I am keenly aware of the fallibility of memory after all this time. To use the cliche, these are "my best recollections".

- Bob

Speaking to the last substantive paragraph, there seem to be no reservations about the Boolean-valued approach expressed on behalf of Tennenbaum in Solovay-Tennenbaum [1971], in footnotes or elsewhere. I was bemused when once Tennenbaum told me that he did not understand their paper. The idea of assigning to a formula in the forcing language a value from a complete Boolean algebra had occurred to Solovay around the time of the collaboration with Tennenbaum; Solovay conveyed the idea to Scott, and by late 1965 they had independently come to the idea of starting with Boolean valued sets from the beginning to do forcing. ${ }^{9}$ Boolean-valued models still held sway when Solovay wrote up SolovayTennenbaum [1971], which was submitted in late 1969.

The several years between proof and write-up allowed for a significant incorporation. Around 1967 Tony Martin observed that "by the same techniques used by the authors to get a model of SH" (Solovay-Tennenbaum [1971:232]) one can establish the consistency of

\footnotetext{
$9 \quad$ See Scott [1985: xivff].
} 
a stronger, focal "axiom", and Solovay named this axiom and incorporated its consistency into the write-up. This was the genesis of Martin's Axiom (MA), independently suggested by Kenneth Kunen and Frederick Rowbottom. Beyond killing all the Suslin trees, one can analogously add "generics" to all countable chain condition forcings if one only has to meet less than continuum many dense sets. With its consistency in hand Martin-Solovay [1970] showed that MA has a wide range of consequences, as it allows for constructions analogous to those from $\mathrm{CH}$. Since then of course, MA has achieved a methodological prominence as a focal "axiom" for relative consistency results.

Solovay-Tennenbaum [1971] is the beginning of genuine, iterated forcing in the following sense. Before their work, the major accomplishments with forcing that had involved the analysis of product forcings had been the work of William Easton [1964][1970] on powers of regular cardinals and the work of Solovay [1965][1970] on Lebesgue measurability. However, in these cases the products were taken of ground model notions of forcing. SolovayTennenbaum [1971] was the first to work with forcings analyzed as iterating one notion of forcing followed by a subsequent one which only occurs in an intermediate generic extension and to show that combinatorial properties like the countable chain condition are preserved. Iterated forcing is part and parcel of modern set theory, and it was born that day in 1965.

Considering the further development of set theory, the sophistication of the algebraic Boolean-valued approach in Solovay-Tennenbaum [1971] had the effect of obscuring the underlying iteration processes. Soon set theorists were back to working entirely with partial orders. William Mitchell [1972] specifically stated the product lemma for the two-step iteration of partial orders, and Richard Laver [1976] cast his iterated forcing even through limit stages in the modern way with sequences of conditions. Around this time I recall Jack Silver being enthused that the consistency of MA can be done with partial orders.

On a final note, the model of Solovay-Tennenbaum [1971] inherently satisfies $\neg$ CH. In a tour de force of forcing for the time, Jensen (cf. Devlin-Johnsbråten [1974]) established the joint consistency Con(ZFC) implies $\mathrm{Con}(\mathrm{ZFC}+\mathrm{CH}+\mathrm{SH})$. This he did by a forcing that adds no new reals, kills Souslin trees by "specializing" them, and is constructed with special inverse limits based on his combinatorial principles derived from $L$. Saharon Shelah once told me that it was trying to come to grips with Jensen's argument that inspired a significant part of his early work with his "proper" forcing. Shelah [1998: V§6] establishes Jensen's result systematically on his proper forcing context.

In an address given at the International Congress of of Logic, Methodology, and Philosophy of Science held at Bucharest in 1971, Alfred Tarski reserved particular praise for Solovay's work on Lebesgue measurability and Solovay and Tennenbaum's work on Suslin's Hypothesis as the most important in set theory since Cohen's work. ${ }^{10}$ In a letter to Gödel of 6 April 1973, Abraham Robinson wrote: "As the outstanding individual achievements in logic since 1963 I would list (in chronological order): 1) The work of Ax-Kochen on pfields. 2) Solovay on measurable sets, and the Tenenbaum-Solovay on Souslin's conjecture. 3) Matiyasevich's solution of Hilbert's 10th problem."

10 This is the recollection of the author, who as a research student attended the conference. 


\section{$\S 4$. Envoi}

My own encounters with Tennenbaum were modest. When I was an instructor at UC Berkeley, my first job, Tennenbaum sauntered into my office one day in the spring of 1976, introduced himself, and promptly made himself comfortable by sitting on the floor. I of course was aware of his work on $\mathrm{SH}$, and in my youthful reckoning of people by their recent accomplishments was somewhat taken aback by all this as well as his general disheveled appearance despite sport-jacket and tie. Anyway, we had a pleasant conversation about my work and prospects in set theory, from which I mainly recall his steady probing for connections and meanings. At one point he asked me for my "pedigree" and I charted my mathematical genealogy on the blackboard: Kanamori - Mathias - Jensen - Hasenjaeger — Scholz. He had heard of Mathias and, of course, Jensen. I explained that Hasenjaeger was a German set theorist who had something to do with the Completeness Theorem, and that through him I was connected to Heinrich Scholz, a theologian who kept mathematical logic alive in Germany between the world wars. For some reason, to this day I can still visualize Tennenbaum's eyes suddenly opening wide and his hands going up to his chin as he pondered the blackboard.

Years later I was touring Montreal with a young family, and I suddenly saw Tennenbaum across the street. It was something about his clothes or the angularity of his walk - he was unmistakable. I was busily shepherding children en train and decided that I could not possibly cross the street and go up to him. But peering out of the corner of my eye, I espied him calmly sauntering up the narrow street, looking this way and that, focusing for a moment on crocuses in a flowerpot. I had recently seen the film Being There, with Peter Sellers in the main role of Chauncy Gardner, and I was pointedly reminded of the final scene, where Gardner is seemingly walking on water, enjoying the water lilies.

\section{REFERENCES}

[1895] GEORG CANTOR, Beiträge zur Begründung der transfiniten Mengenlehre. I, Mathematische Annalen 46, 481-512; translated in [1915] below.

[1915] GEORG CANTOR, Contributions to the Founding of the Theory of Transfinite Numbers, with introduction and notes by Philip E.B. Jourdain, Open Court, Chicago; reprinted by Dover, New York 1965.

[1995] JOSEPH W. DAUBEN, Abraham Robinson. The Creation of Nonstandard Analysis. A Personal and Mathematical Journey, Princeton University Press, Princeton.

[1974] KEITH J. DEVLIN and HAVÅRD JOHNSBRÅTEN, The Souslin Problem, Lecture Notes in Mathematics \#405, Springer-Verlag, Berlin.

[1964] WILLIAM B. EASTON, Powers of regular cardinals, Ph.D. thesis, Princeton University; abstracted as: Proper classes of generic sets, Notices of the American Mathematical Society 11 (1964), 205; published in abridged form as [1970] below.

[1970] WILLIAM B. EASTON, Powers of regular cardinals, Annals of Mathematical Logic $1,139-178$.

[1967] THOMAS J. JECH, Non-provability of Souslin's Hypothesis, Commentationes mathematicae Universitatis Carolinae 8, 291-305. 
[1968] RONALD B. JENSEN, Souslin's Hypothesis is incompatible with $V=L$, Notices of the American Mathematical Society 16, 935.

[1972] RONALD B. JENSEN, The fine structure of the constructible hierarchy, Annals of Mathematical Logic 4, 229-308.

[1995] AKIHIRO KANAMORI, The emergence of descriptive set theory, in: Jaakko Hintikka (editor), From Dedekind to Gödel: Essays on the Development of the foundations of Mathematics, Synthese Library volume 251, Kluwer, Dordrecht.

[1980] KAZIMIERZ KURATOWSKI, A Half-Century of Polish Mathematics. Remembrances and Reflections, Oxford, Pergamon Press.

[1935] DJURO R. KUREPA, Ensembles ordonnés et ramifiés, Thèse, Paris; published as Publications mathématiques de l'Université de Belgrade 4, 1-138.

[1936] DJURO R. KUREPA, H'hypothèse de ramification, Comptes rendues hebdomadaires des séances de l'Académie des Sciences, Paris 202, 185-187.

[1968] MARY GRACE KUZAWA, Modern Mathematics. The Genesis of a School in Poland, New Haven, College \& University Press.

[1976] RICHARD LAVER, On the consistency of Borel's conjecture, Acta Mathematica 137, 151-169.

[1917] NIKOLAI LUZIN, Sur la classification de M. Baire, Comptes rendues hebdomadaires des séances de l'Académie des Sciences, Paris 164, 91-94.

[1970] DONALD A. MARTIN and ROBERT M. SOLOVAY, Internal Cohen extensions, Annals of Mathematical Logic 2, 143-178.

[1972] WILLIAM MITCHELL, Aronszajn trees and the independence of the transfer property, Annals of Mathematical Logic 5, 21-46.

[1943] EDWIN W. MILLER, A note on Souslin's Problem, American Journal of Mathematics $65,673-678$.

[1955] MARY ELLEN RUDIN, Countable paracompactness and Souslin's Problem, Canadian Journal of Mathematics 7, 543-547.

[1969] MARY ELLEN RUDIN, Souslin's Conjecture, American Mathematical Monthly 76, 1113-1119.

[1985] DANA S. SCOTT, Foreword to John L. Bell, Boolean-valued Models and Independence Proofs in Set Theory, Second edition, Oxford Logic Guides \#12, Oxford University Press, Oxford.

[1984] SAHARON SHELAH, Can you take Solovay's inaccessible away? Israel Journal of Mathematics 48, 1-47.

[1998] SAHARON SHELAH, Proper and Improper Forcing, Springer, Berlin.

[1948] WACŁAW SIERPIŃSKI, Sur un problème de la théorie générale des ensembles equivalent ua problème de Souslin, Fundamenta Mathematicae 35, 165-174.

[1965] ROBERT M. SOLOVAY, The measure problem (abstract), Notices of the American Mathematical Society 12, 217.

[1970] ROBERT M. SOLOVAY, A model of set theory in which every set of reals is Lebesgue measurable, Annals of Mathematics 92, 1-56. 
[1971] ROBERT M. SOLOVAY and STANLEY TENNENBAUM, Iterated Cohen extensions and Souslin's problem, Annals of Mathematics (2)94, 201-245.

[1917] MIKHAIL Ya. SUSLIN, Sur une définition des ensembles mesurables B sans nombres transfinis, Comptes rendues hebdomadaires des séances de l'Académie des Sciences, Paris $164,88-91$.

[1920] MIKHAIL Ya. SUSLIN, Problème 3, Fundamenta Mathematicae 1, 223.

[1968] STANLEY TENNENBAUM, Souslin's Problem, Proceedings of the National Academy of Sciences of the United States of America 59, 60-63.

[1984] STEVO TODORČEVIĆ, Trees and linearly ordered sets, in: Kenneth Kunen and Jerry E. Vaughan (editors), Handbook of Set-Theoretic Topology, North-Holland, Amsterdam, 235-293.

[1954] ALEXANDER S. YESENIN-VOLPIN, Nedokazyemost gipotezy Suslina bez pomoshchi aksiomy vybora v systeme aksiom Bernaysa-Mostovskogo, Doklady Akademii Nauk SSSR 96, 9-12; translated in [1963] below.

[1963] ALEXANDER S. YESENIN-VOLPIN, The unprovability of Suslin's Hypothesis without the aid of the Axiom of Choice in the Bernays-Mostowski axiom system, American Mathematical Society Translations (2)23, 83-88.
Akihiro Kanamori
Department of Mathematics
Boston University
Boston, Massachusetts 02215
United States of America
email: aki@math.bu.edu 